\section{Mortality of patients with COPD participating in chronic disease management programmes: a happy end?}

\author{
ABSTRACT \\ Background Concerns about increased \\ mortality could question the role of COPD \\ chronic disease management (CDM) \\ programmes. We aimed at extending a recent \\ Cochrane review to assess the effects of CDM \\ on mortality in patients with COPD. \\ Methods Mortality data were available for \\ 25 out of 29 trials identified in a COPD \\ integrated care systematic review. Meta- \\ analysis using random-effects models was \\ performed, followed by subgroup analyses \\ according to study length (3-12 months vs \\ $>12$ months), main intervention component \\ (exercise, self-management, structured follow- \\ up) and use of an action plan.
}

Results The meta-analysis showed no impact of CDM on mortality (pooled OR: 1.00, 95\% $\mathrm{Cl} 0.79$ to 1.28 ).

Conclusions These results do not suggest that CDM programmes expose patients with COPD to excessive mortality risk.

For more than two decades, chronic disease management (CDM) initiatives have been developed and implemented in many countries. CDM aims at reorganising healthcare systems and medical treatment to address the increasing burden of chronic diseases and provide solutions to organisational, medical and economical problems. A recent Cochrane systematic review showed that in patients with COPD, CDM significantly improved health-related quality of life as well as exercise tolerance (6-min walking test; 6MWT) and decreased the risk of hospitalisation. ${ }^{1}$ While updating non-Cochrane systematic reviews published in the mid-2000s and conducting a methodologically sound and rigorous Cochrane systematic review, Kruis et al confirmed previous trends in outcomes, indeed. Unfortunately, analyses of secondary outcomes, such as mortality, were not performed comprehensively enough from our perspective. In fact, out of 26 studies included in the review and 3 studies awaiting assessment, ${ }^{2-4}$ only data from the five randomised controlled trials (RCTs) explicitly reporting mortality as an outcome were considered in the mortality meta-analysis. Mortality results are of crucial importance for those interested in CDM development and implementation in the field, particularly since Fan's publication in 2012. ${ }^{3}$ In the latter study in fact, health benefits from such a programme were counterbalanced by an unexpected and still unexplained significantly higher mortality in patients with COPD participating in a comprehensive care programme.

The aim of our targeted analyses was to assess mortality of patients with COPD participating in CDM programmes using data from studies included in Kruis' recent Cochrane review. ${ }^{1}$ Because all-cause mortality is an indisputable outcome, even if not a targeted study event, we considered all 29
RCTs described in Kruis' review: 26 RCTs meeting Kruis' operational definition of CDM ( $\geq 2$ different types of healthcare professionals actively involved in patients care, $\geq 2$ intervention components, $\geq 3$ months' duration) and included in the analyses, as well as three studies identified as 'awaiting assessment'. ${ }^{1}$ We reviewed the three latter studies and considered them to meet inclusion and exclusion criteria of the systematic review; one of the three was the Fan's RCT. $^{3}$ Mortality data were available for 25 of the 29 eligible studies described in Kruis' review. Baseline number of patients included in both CDM and control groups, number of deaths in each group during studies' periods as well as studies' length, main intervention component and use of action plan, were extracted. We conducted a random-effects meta-analysis, followed by subgroup analyses according to study length (3-12 months vs >12 months), main intervention component (exercise, self-management, structured follow-up) and specific use of an action plan in the intervention.

Our enriched meta-analysis showed no impact of CDM on overall mortality (25 studies; pooled OR: $1.00,95 \%$ CI 0.79 to 1.28; figure 1). There was little evidence for heterogeneity $\left(\mathrm{I}^{2}\right.$ 19\%) and only discrete asymmetry as assessed by a funnel plot. All subgroup analyses showed nonsignificant pooled ORs. As event rates were low, we also computed the unweighted estimate of the pooled OR after adding 0.5 to observed frequencies of all studies, as

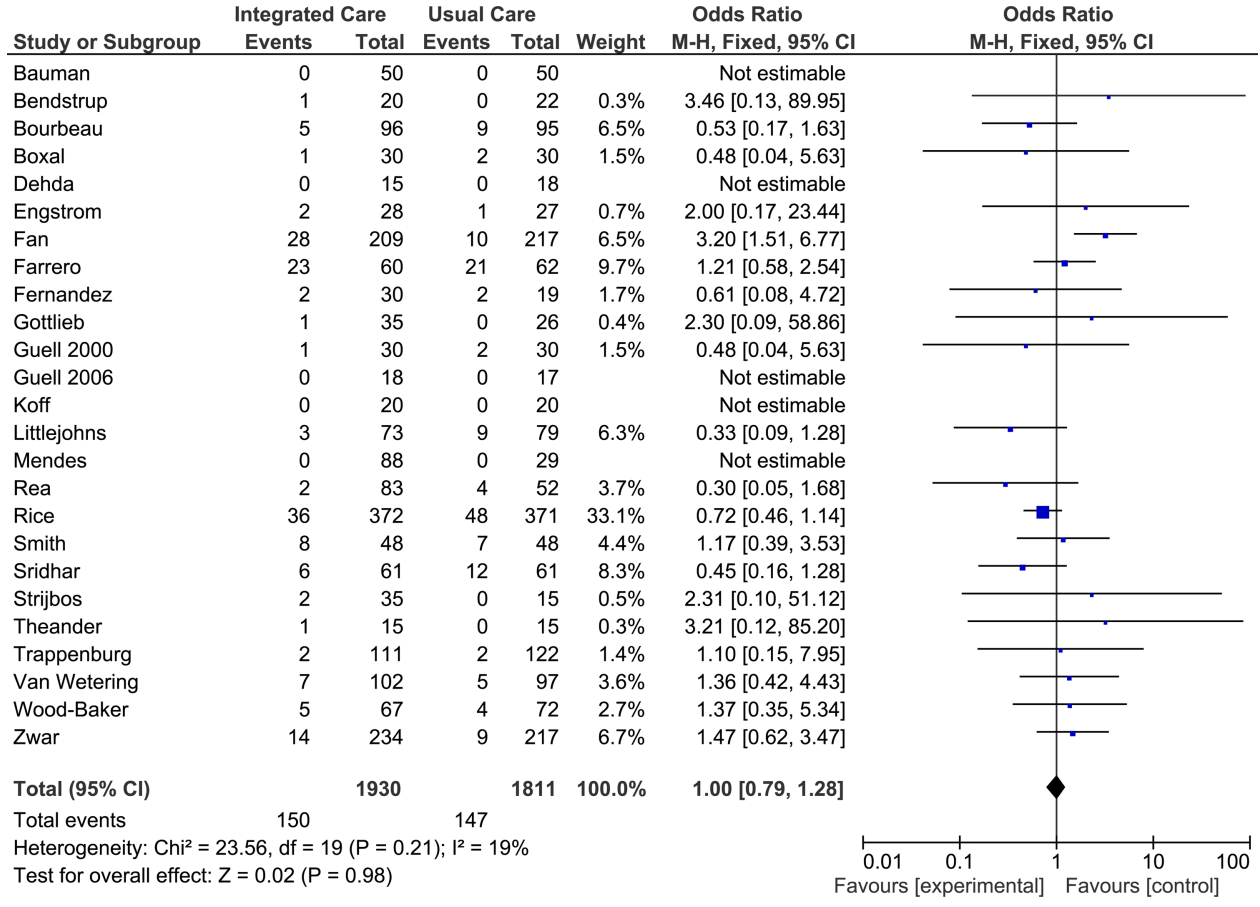

Figure 1 Overall mortality forest plot. 
suggested by Bhaumil et al. ${ }^{5}$ This methodology allows the inclusion of zero total events and has been shown to perform better than the traditional DerSimonian and Laird approach in those situations. Results were however similar to the standard ones (pooled OR $=1.07$ 95\% CI 0.58 to 1.84 , between-study variance $\tau^{2}=0.4$ ). Varying the between-study variance $\left(0.08 \leq \tau^{2} \leq 0.6\right)$ did not alter the results, thereby illustrating their robustness.

Results of this enriched meta-analysis do not suggest that complex interventions such as CDM expose patients with COPD to excessive mortality risk, as feared by a recent $\mathrm{RCT}^{3}$

\section{Peytremann-Bridevaux, ${ }^{1}$ P Taffe, ${ }^{1}$ B Burnand, ${ }^{1}$ P O Bridevaux, ${ }^{2,3}$ M A Puhan ${ }^{4}$}

${ }^{1}$ Healthcare Evaluation Unit, Institute of Social and Preventive Medicine, Lausanne University Hospital, Lausanne, Switzerland

${ }^{2}$ Centre Hospitalier du Valais Romand, Sion, Switzerland ${ }^{3}$ Division of Pulmonary Medicine, Geneva University Hospital, Geneva, Switzerland
${ }^{4}$ Institute of Social and Preventive Medicine, University of Zurich, Zurich, Switzerland

Correspondence to Professor Isabelle PeytremannBridevaux, Institute of Social and Preventive Medicine, 10 route de la Corniche, Lausanne 1010, Switzerland; Isabelle. Peytremann-Bridevaux@chuv.ch

Contributors IP-B was at the origin of the project, extracted data, conducted analysis and wrote the manuscript. PT performed statistical analysis and participated in the writing of the manuscript. POB and MAP participated in the elaboration of the project, extracted data and participated in the writing of the manuscript. BB participated in the elaboration of the project and provided insightful comments to the manuscript. All authors read and approved the final version of the manuscript.

Competing interests IP-B is supported by a grant from the Swiss National Science Foundation (PROSPER no. 32333B-123817 and no. 32333B-139789) and by the Swiss School of Public Health+ (Assistant Professorship grant).

Provenance and peer review Not commissioned; externally peer reviewed.

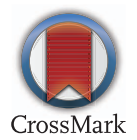

To cite Peytremann-Bridevaux I, Taffe P, Burnand B, et al. Thorax 2014;69:865-866.

Received 11 December 2013

Revised 10 March 2014

Accepted 21 March 2014

Published Online First 9 April 2014

Thorax 2014;69:865-866.

doi:10.1136/thoraxjnl-2013-204983

\section{REFERENCES}

1 Kruis AL, Smidt N, Assendelft WJJ, et al. Integrated disease management interventions for patients with chronic obstructive pulmonary diseases. Cochrane Database Syst Rev 2013;10:CD009437.

2 Baumann HJ, Kluge S, Rummel K, et al. Low intensity, long-term outpatient rehabilitation in COPD: a randomised controlled trial. Resp Res 2012;13:86.

3 Fan VS, Gaziano JM, Lew R, et al. A comprehensive care management program to prevent chronic obstructive pulmonary disease hospitalizations: a randomized, controlled trial. Ann Intern Med 2012;156:673-83.

4 Zwar NA, Hermiz O, Comino E, et al. Care of patients with a diagnosis of chronic obstructive pulmonary disease: a cluster randomised controlled trial. Med J Austr 2012;197:394-98.

5 Bhaumik DK, Amatya A, Normand SL, et al. Metaanalysis of rare binary adverse event data. J Am Stat Assoc 2012;107:555-67. 